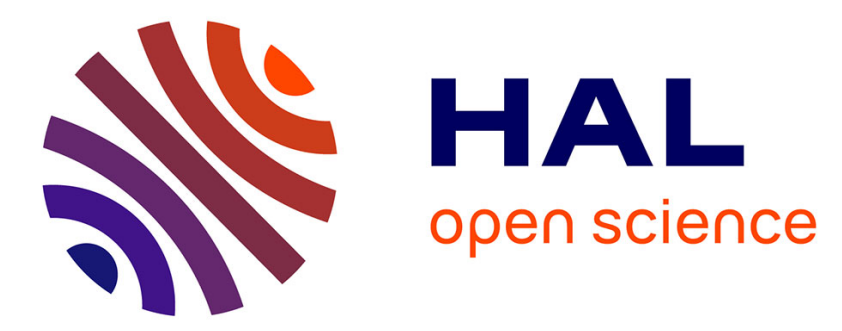

\title{
Performance of vehicle platooning under different V2X relaying methods
}

\author{
Tiago R Gonçalves, Vineeth S Varma, Salah Eddine Elayoubi
}

\section{To cite this version:}

Tiago R Gonçalves, Vineeth S Varma, Salah Eddine Elayoubi. Performance of vehicle platooning under different V2X relaying methods. IEEE PIMRC 2021, Sep 2021, Helsinki (virtual), Finland. hal-03295946

\section{HAL Id: hal-03295946 \\ https://hal.science/hal-03295946}

Submitted on 22 Jul 2021

HAL is a multi-disciplinary open access archive for the deposit and dissemination of scientific research documents, whether they are published or not. The documents may come from teaching and research institutions in France or abroad, or from public or private research centers.
L'archive ouverte pluridisciplinaire HAL, est destinée au dépôt et à la diffusion de documents scientifiques de niveau recherche, publiés ou non, émanant des établissements d'enseignement et de recherche français ou étrangers, des laboratoires publics ou privés. 


\section{Performance of vehicle platooning under different $\mathrm{V} 2 \mathrm{X}$ relaying methods}

\author{
Tiago R. Gonçalves \\ Université Paris-Saclay \\ CentraleSupélec - CNRS - L2S \\ Gif-sur-Yvette, France \\ tiago.rochagoncalves@centralesupelec.fr
}

\author{
Vineeth S. Varma \\ Université de Lorraine \\ CRAN UMR 7039 and the CNRS \\ Nancy, France \\ vineeth.satheeskumar-varma@univ-lorraine.fr
}

\author{
Salah E. Elayoubi \\ Université Paris-Saclay \\ CentraleSupélec - CNRS - L2S \\ Gif-sur-Yvette, France \\ salaheddine.elayoubi@centralesupelec.fr
}

\begin{abstract}
We study in this paper the design of the vehicleto-everything (V2X) network for vehicle platooning scenarios. We focus on the impact of packet relaying using Road Side Units (RSU) on the application-level performance, namely the inter-vehicle distance in the platoon. The RSU extends the coverage range of the platoon leader and allows handling the well-known Predicted Cooperative Adaptive Cruise Control (PCACC) scheme. We develop a Markov model for evaluating the performances of the different communication links (inter-vehicle and vehicle-to-RSU), using licensed or unlicensed spectrum. We then develop a cross-layer approach that adapts the application layer (PCACC control parameters) to the observed Medium Access Layer (MAC) performance. The simulations results prove that relaying allows to greatly reduce both the communication link failures and the intervehicular distances while introducing minimal delay in the system. Furthermore, even if using licensed spectrum for the relay link it drastically reduces the Packet Error Rate (PER) compared to the unlicensed spectrum case, the robustness of the application layer scheme makes the latter case viable for the considered scenario.

Index Terms-Vehicle platoons, relay, cooperative adaptive
\end{abstract} cruise control (CACC), wireless communication.

\section{INTRODUCTION}

Advanced Driver-Assistance Systems (ADAS) are intelligent systems located inside the vehicle and aiming at increasing driver's safety and comfort, reducing emissions and fuel consumption, and increasing traffic-flow. Our particular interest is in the platooning use case that takes advantage of the particular distribution of a convoy in order to increase road capacity and to decrease fuel consumption, by gathering vehicles close together in order to reduce the air resistance of the platoon's members. However, the feasibility and the deployment of platoons relies on the reliable and fast exchange of information between vehicles as it allows taking control actions based on the most up-to-date information about the road and traffic status. Nevertheless, such an exchange of information occurs over an unreliable wireless communication channel subject to inherent characteristics such as latency and packet loss.

The communication technology adopted and evaluated in this paper is the IEEE $802.11 \mathrm{p}$, based on carrier-sensing multiple access with collision avoidance (CSMA/CA) protocol, and is based on the well known Bianchi model [1], with extensions that allow considering specificity of platooning systems: The presence of a large number of pointto-point V2V links, coexisting with a broadcast relaying link that conveys the leader's packets to the rest of the platoon. Our model also integrates the impact of radio errors resulting from the physical layer (link performance) within the Medium Access Control (MAC) layer model (packet collision estimation).

We focus in our work on the performance metrics of the end service (inter-vehicular distance of the platoon) when evaluating communication schemes. While the baseline scheme corresponds to the use of V2V links only, we introduce the impact of relaying through Road Side Units (RSU), using licensed or unlicensed spectrum. We first propose a novel model for the channel access schemes with the presence of relaying links through the RSU. In addition to that, we introduced a dynamic control mechanism where some of the parameters of the PCACC controller are adapted based on the expected quality of the radio system. The main contributions of this paper are summarized as follows.

- Proposition of a new analytical model of the network performance, with and without relaying. These models integrate the impacts of link and system levels in both licensed and unlicensed spectrum cases.

- Online adaptation of the platoon control parameters based on the observed communication link quality and on the results of the offline optimization.

- Assess the performance of the platoon under the different communication solutions and subject to difficult traffic jamming environment on the road.

- Compare the communication solutions from an endservice perspective (platoon efficiency), and not only from network-related Key Performance Indicators (KPI) as it is usually the case.

\section{RELATED WORK}

As of the literature on V2X communications for platooning, a considerable number of papers have proposed different approaches in order to extend the coverage range of the leader of the platoon to other platoon members. [2] proposed an enhancement in the relay election procedure by considering that the best relay is the node from which every node in the targeted part of the destination area is able to receive. A recent evaluation on side-link relay for platooning was done by [3] where the authors proposed two relay schemes that use geographic location information. Inspired by Bianchi's 2-D Markov chain [1], the authors in [4] proposed a platoon-based cooperative retransmission scheme by formulating a 4-D Markov chain so that one sender can retransmit blocks for its neighbors within the same platoon in case of a previous transmission error. In opposition to these works, we do not aim at proposing new relaying schemes for platooning but use the existing relaying 


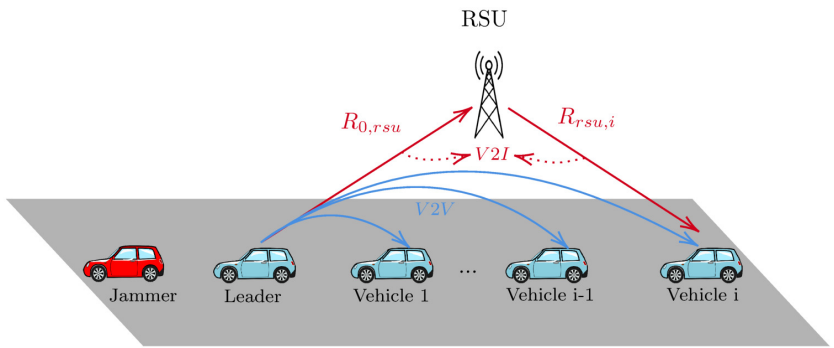

Fig. 1. Disposal of a platoon with V2V and V2I communication technologies.

methods through RSU to study the performance from an end-user perspective.

Another important contribution of our paper is related to the joint communication/control approach. Several works evaluate the performance of platooning under different communication approaches. For instance, [5] used the 802.11p technology to evaluate the communication performance under a CACC controller in platoons. Likewise, [6] has adopted both wireless technologies (802.11p and C-V2X) and compared their performances in terms of the intervehicular distance of the platoon. [7] proposed a control strategy for graceful degradation based on estimating the preceding vehicle's acceleration in case of packet losses, but it mainly deals with extreme cases like complete link failure or lack of a wireless device on one of the vehicles. [8] suggested different information management algorithms including one with a dynamical control parameter where they simply suggest a lower bound value for it. Our proposed dynamic controller goes far beyond a graceful degradation framework that switches to a completely local controller or a simple performance assessment under errors, as it continuously adapts to the link quality while preserving the robustness. Note that in a previous work [9], we proposed a centralized design for the controller under a simple radio model based on V2V only, while in this paper we develop a joint communication/control model that considers sophisticated radio link and system models and in the presence of RSU relaying.

\section{SYSTEM MODEL}

This paper considers a longitudinal platoon (blue vehicles), as depicted in Fig. 1, of size $N$ arranged as 1 platoon leader and $N-1$ platoon members. The vehicles in all other lanes are not necessarily in platoons, and a jammer precedes (in red) the platoon leader. In order to stabilize the platoon under the presence of the jammer, while reducing the distance between the platoon members, the platoon leader communicates data about its acceleration and velocity to all the members of the platoon, as does also each vehicle to its following one. If packets are lost or delayed, the platoon performance may be adversely affected. Therefore, the communication links reliability is imperative to the deployment of controlled platoons. We introduce in this section a brief overview of the system with the proposed dynamic control, and the considered communication scenarios specific to platooning.

\section{A. Platoon controller}

We here describe briefly the application level control schemes for the platooning scenario. A thorough under- standing of the dynamics of the controller is indeed essential for the design of relevant communication schemes.

1) Predictive Cooperative Adaptive Cruise Control: PCACC, introduced in [10], implies that the control effort, the desired acceleration, of the leader $\left(\ddot{x}_{0 \_} d e s\right)$ and of the preceding vehicle $\left(\ddot{x}_{(i-1) \_d e s}\right)$ are available to the following vehicle and its control law is given by

$$
\begin{aligned}
a_{i_{-} d e s}=\ddot{x}_{i_{-} d e s} & =(1-C) \ddot{x}_{(i-1) \_d e s}+C \ddot{x}_{0_{-} d e s} \\
& -\left(2 \xi-C\left(\xi+\sqrt{\xi^{2}-1}\right)\right) \omega_{n} \dot{\epsilon}_{i} \\
& -\left(\xi+\sqrt{\xi^{2}-1}\right) \omega_{n} C\left(\dot{x}_{i}-\dot{x}_{0}\right)-\omega_{n}^{2} \epsilon_{i}
\end{aligned}
$$

where

$$
\begin{aligned}
& \epsilon_{i}=x_{i}-x_{i-1}+L_{i}+D_{\text {des }} \\
& \dot{\epsilon}_{i}=\dot{x}_{i}-\dot{x}_{i-1} .
\end{aligned}
$$

$L_{i}$ is the length of the vehicle and $D_{d e s}$ is the desired inter-vehicular distance that we want to minimize. The subscript $i$ is the vehicle platoon member index where $i \in\{1, \cdots, N-1\}$ and 0 the platoon leader index. The control parameters to be tuned are $C, \xi$ and $\omega_{n}$. The parameter $C$ takes on values $0 \leq C<1$ and is responsible to weigh the contribution of the leader's speed and acceleration. $\xi$ is the controller damping ratio and $\omega_{n}$ is the controller bandwidth. Therefore, the most up-to-date values of the $\left(\ddot{x}_{0 \_d e s}, \dot{x}_{0}, \ddot{x}_{(i-1) \_d e s}\right)$ in (1) are subject to wireless inherent characteristics such as packet dropouts or delay.

2) Vehicle dynamics: The vehicle dynamics is modeled as first-order low pass filter due to the actuator lag. So the transfer function is modeled in the frequency-domain as

$$
G(s)=\frac{A_{i}(s)}{A_{i_{-} d e s}(s)}=\frac{1}{\tau s+1}
$$

where $\tau$ is the time constant of the first-order low pass filter. $A_{i}$ is the output, which can be interpreted as the actual vehicle acceleration, whereas $A_{i_{-} d e s}$ is the vehicle input, which can be seen as the desired acceleration. Note that, $\cdot(s)$ denotes the Laplace transform of the corresponding timedomain variable. The idea is to approximate the dynamics of the throttle body and vehicle inertia in order to avoid instantaneous response. In this paper, we assumed a lag of $\tau=0.5 \mathrm{~s}$ as in [11] in order to be conservative.

3) Proposed adaptive control scheme based on PCACC: In contrast to existing works that assume a fixed control strategy, we adopt an approach that adapts the control parameters based on the communication link quality, as introduced in our previous work [9]. In particular, among the control inputs, the parameter $D_{d e s}$ has a substantial impact on the performance of the system. As mentioned before, $D_{d e s}$ is the desired inter-vehicular distance that we want to set, but due to actuator lag and delay in the process it does not correspond to the actual average inter-vehicular distance $\left(D_{\text {avg }}\right)$. Therefore, in this work we conduct an offline heuristic optimization to determine the best control parameter $D_{\text {des }}$ in terms of minimum average inter-vehicular distance without collisions, for any treated communication platoon scenario. 


\section{B. Communication mechanisms for platooning}

In order for the platoon control to be efficient, there is a need for a reliable exchange of information between neighboring vehicles and from the leader to the rest of the platoon. We introduce in this section the communication solutions, as outlined in Fig. 1, before moving to the performance evaluation model.

1) Baseline scheme with V2V communications only: In this baseline, vehicles use direct communication links based on IEEE $802.11 \mathrm{p}$ on the unlicensed spectrum, and thus applying CSMA/CA mechanism. There are three types of links, as follows:

- Vehicle to neighbor links, where each vehicle conveys its acceleration and velocity to its preceding one. For a platoon of $N$ vehicles, there are $N-1$ such links. We denote by $(i, i+1)$ the link between vehicle number $i$ and its preceding. Following the CSMA/CA mechanism, a packet on this link is repeatedly transmitted until an ACK is received from vehicle $i+1$, or the maximum number of transmissions, say $m$ is reached.

- Broadcast link, where the platoon leader communicates its information to the rest of the platoon. As there is no native broadcast link design in CSMA/CA, it cannot be expected that an $\mathrm{ACK}$ is received from each vehicle. The simplest scheme is to consider that the leader sends its packets to vehicle 1 (on the link $(0,1)$ defined above) and expect ACK for vehicle 1 only. Retransmission mechanism is thus subject to the channel quality of link $(0,1)$. However, this communication is overheard by other vehicles in the platoon that may be able to decode it. We call brodcast link as seen by vehicle $i$, link $(0, i)$.

- Outside links, that correspond to an interfering link that does not belong to the platoon. We consider $M$ such links and model them as interference.

2) RSU relaying of the leader's packets: Looking back at the control mechanism of equation (1), the $(i, i+1)$ links convey local control information that is weighted by parameter $(1-C)$, while link $(0, i)$ is responsible for carrying information of the leader, weighted by parameter $C$. Knowing that the distance on link $(0, i)$ is generally large and that it is subject to larger shadowing (because of the existence of cars in-between the leader and the vehicle $i$ ), it is of utmost importance to enhance its quality. We adopt in this paper relaying through RSU as a solution for the broadcast link, where the RSU relays the packet received from the leader, in a broadcast manner to all other vehicles. We consider two flavors of relaying:

- Relaying on licensed spectrum, using cellular C-V2X technology. This scheme has the advantage of using a dedicated spectrum in a scheduled mode. There are two additional links in this case to model: The uplink $(0, R S U)$ on which the leader sends its packet and the downlink $(R S U, i)$ where the RSU broadcasts the information to other cars. Again, we do not consider a feedback mechanisms on the broadcast link.

- Relaying on unlicensed spectrum. In this case, the RSU is considered as an additional node using the IEEE $802.11 p$ system; it overhears the packet sent by the leader to vehicle 1 (let $(0, R S U)$ be this link), and then

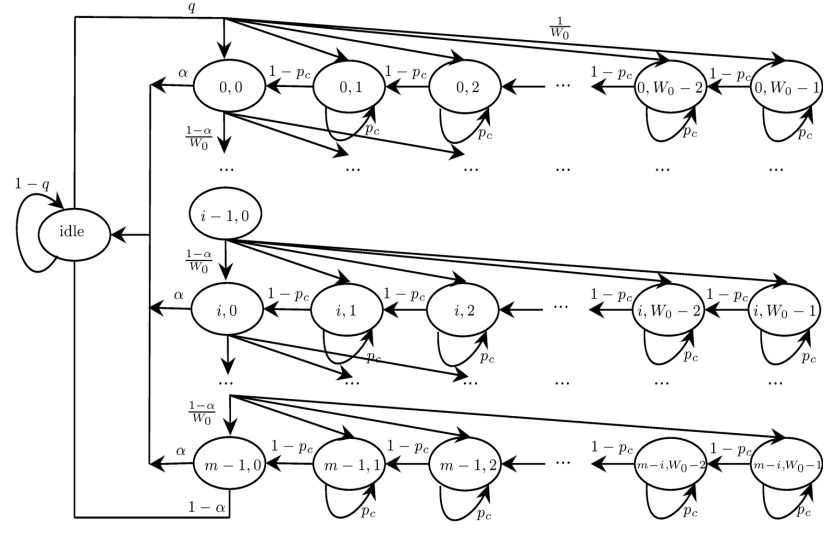

Fig. 2. Proposed Markov chain for baseline scheme.

retransmits it in a broadcast manner, without expecting feedback.

Note that since the RSU acts as a broadcast relay, it does not expect or receive any feedback, and therefore, does not attempt re-transmissions even if its packets are lost. The main advantage of the RSU is having a direct line of sight link with both the leader and all the other vehicles in the platoon.

3) Link and system performance metrics: Before moving to the performance model, we identify the main performance metrics on the different links. We differentiate between link and system levels.

At link level, performance is characterized by the average Packet Error Rate (PER), i.e. the probability that a particular transmission of a packet fails, due to fast fading and to interference from other links. This PER takes two different values when the packet is conveyed alone, compared to the collision case when it is transmitted on an occupied channel. Let $f_{0,(k, l)}$ and $f_{c,(k, l)}$ be the PER for the collision-free and collision case, respectively, for links $(k, l)$ defined above $(k$ and $l \in\{R S U, 0, \ldots, N-1\}$.

At system level, the main performance metric is the packet loss, that incorporates the PER on the link level, but also takes into account the $m$ possible retransmissions and the CSMA/CA mechanism. We develop in the next section a performance model on the system level (channel access) that takes into account the link level metrics.

\section{Performance MODEL}

In this section, we propose an analytical model adapted to different platooning scenarios, and integrating both link and channel access levels. We first devise a Markov chain to model the CSMA/CA protocol with retransmissions for point-to-point V2V links. Next, we introduce the broadcast link analysis and derive the probability that a given vehicle, numbered $i$, receives the packets of the leader. We finally extend the model to consider the relay case for both licensed and unlicensed spectrum.

\section{A. Packet loss probability for V2V transmissions}

We model the CSMA/CA channel access procedure using a discrete time Markov chain as illustrated in Fig. 2. However, different from [1], we consider, in addition to losses due to collisions between packets, losses that are due to imperfections on the radio channel. 
1) Vehicle to neighbor channel: At each transmission attempt, and focusing on link $(k, l)$, the packet is correctly decoded with probability

$$
\alpha_{(k, l)}=\left(1-p_{c}\right) \cdot\left(1-f_{0,(k, l)}\right)+p_{c} \cdot\left(1-f_{c,(k, l)}\right)
$$

$p_{c}$ is the probability that the channel is busy during a slot (collision probability), $f_{0}$ is the probability of loss without collision, and $f_{c}$ is the probability of loss with collision, introduced previously and computed in the numerical applications by an SINR (Signal to Interference and Noise Ratio) computation on a vehicular channel. We drop in the following the link identification $(k, l)$ for convenience, except when needed.

In order to capture, the bursty nature of the traffic where small packets are generated by each vehicle following a Poisson process of intensity $\lambda$, we have included one inactive state in the Markov chain to model the probability to remains idle on a slot, taken equal to a packet duration $T$. This is given by $1-q=e^{-\lambda T}$.

To calculate the loss, we have to calculate the stationary probabilities of the states. Define the Backoff Timer (BOT) as a randomly number chosen in the range $\left(0, W_{0}-1\right)$ where $W_{0}$ is the contention window (CS) size ${ }^{1}$. Next, define the Backoff Stage (BOS), as the stage attempt to transmit the packet. Let $s(t)$ be the stochastic process representing the $\operatorname{BOS}\{0, \ldots, m-1\}$ and $\Pi(t)$ representing BOT at time $t$. Let $\Pi_{i, j}=\lim _{t \rightarrow \infty} P\{s(t)=i, \Pi(t)=j\}, i \in$ $\{0, m-1\}, j \in\left\{0, W_{0}-1\right\}$ be the stationary distribution of the chain. Therefore, we can recursively calculate the probability of states for the first backoff stage $i=0$ and for any timer $2 \leq j \leq W_{0}-1$, given by

$$
\Pi_{0, W_{0}-j}=\frac{j q}{\left(1-p_{c}\right) W_{0}} \Pi_{i d l e} .
$$

Now, evaluating the last state (timer expiration $j=W_{0}$ ) for the first stage $(i=0)$, we have the following

$$
\Pi_{0,0}=\frac{q}{W_{0}} \Pi_{i d l e}+\left(1-p_{c}\right) \Pi_{0,1}=q \Pi_{i d l e} .
$$

Therefore, taking into account the probability of success transmission introduced in (5), we can define the following

$\Pi_{1, W_{0}-1}=\frac{(1-\alpha)}{W_{0}} \Pi_{0,0}+p_{c} \Pi_{1, W_{0}-1}=\frac{(1-\alpha) q}{\left(1-p_{c}\right) W_{0}} \Pi_{i d l e}$.

Recursively, we can calculate the last states probabilities $\left(j=W_{0}\right)$ for any backoff stage as

$$
\Pi_{i, 0}=(1-\alpha)^{i} q \Pi_{i d l e},
$$

for $0<i<m-1$. Therefore, the probability of stationary states are given by

$$
\Pi_{i, W_{0}-j}=\frac{j q(1-\alpha)^{i}}{\left(1-p_{c}\right) W_{0}} \Pi_{i d l e},
$$

for $0 \leq i \leq m-1$ and $1 \leq j \leq W_{0}-1$. Moreover, the steady-state probabilities must satisfy the normalization condition $\Pi^{\prime} \Pi=1$, that yields

$$
\Pi_{i d l e}=\left[1+\frac{q\left(1-(1-\alpha)^{m}\right)}{\alpha} \cdot\left(1+\frac{W_{0}-1}{2\left(1-p_{c}\right)}\right)\right]^{-1} \text {. }
$$

\footnotetext{
${ }^{1}$ The CW may change from one stage to another, but we adopt here, without loss of generality, a constant $\mathrm{CW}$, as advocated for delay-sensitive services using LBT cat3.
}

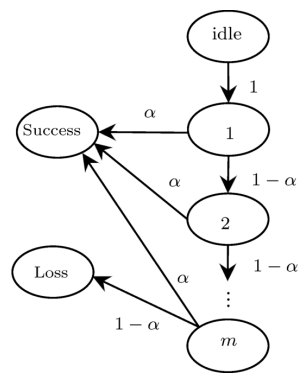

Fig. 3. Proposed Markov chain for leader-follower link.

The probability that a packet is lost on link $(i, i+1)$, despite the $m$ possible retransmissions, is then computed by:

$$
L_{i, i+1}=\left(1-\alpha_{(i, i+1)}\right)^{m}, \quad i \in\{0, \ldots, N-1\}
$$

The loss depends on the probability of finding the channel occupied during a slot, that we will compute using a fixed point approach.

2) Fixed point analysis: The probability of a slot being busy is computed as the probability that at least one of the competing transmitters is active. We consider $N$ vehicles within the platoon, and $M$ external vehicles that do not belong to the platoon but generate nevertheless packets. We can thus compute the probability of busy channel as

$$
p_{c}=1-\left(1-\tau_{p}\right)^{N-1}\left(1-\tau_{e}\right)^{M},
$$

where $\tau_{p}$ (rep. $\tau_{e}$ ) is the probability of trying to transmit for platoon and external vehicles, respectively. Such a probability of transmission can be computed by:

$$
\tau=\sum_{i=0}^{m-1} \Pi_{i, 0}=\frac{\left(1-(1-\alpha)^{m}\right)}{\alpha} q \Pi_{i d l e} .
$$

With the adequate link decoding probability $\alpha$. For platoon vehicles, $\alpha_{(i, i+1)}$ is used, while for external vehicles, the same Markov model as in section III-A1 can be used, replacing the PER in equation (5) by the PER corresponding to a typical distance on a non-platoon link.

The channel occupation probability $p_{c}$ can thus be obtained using a fixed point analysis that solves the set of equations $(11,13,14)$.

3) Broadcast links: We make use of the previously developed Markov chain and focus on link $(0,1)$ that models the medium access between leader-follower. The key point here is that, for other platoon members numbered $i \in\{2, \cdots, N-1\}$, this link is seen as a broadcast link where each packet is repeated $n \leq m$ times, until the packet is correctly received by vehicle 1 . The number of transmissions on the leader-follower link is modeled using the modified Markov chain of Fig. 3, where two absorbing states are added, modeling the loss and success of a packet, starting from the idle state. Here, each stage is contracted within one aggregate state for visibility. The probability of transmitting more than $n$ times is the hitting probability of stage number $n$ starting from the idle state. The probability of transmitting a packet exactly $n$ times is thus computed by:

$$
P_{n}= \begin{cases}\alpha_{(0,1)}\left(1-\alpha_{(0,1)}\right)^{n-1} & \text { for } n<m \\ \left(1-\alpha_{(0,1)}\right)^{m-1} & \text { for } n=m\end{cases}
$$


The second component is the probability for the leader message to fail at the receiver vehicle $i$, considering packet collisions and path loss, as in equation (5).

Therefore, considering broadcast link $(0, i)$, its probability of loss is:

$$
L_{(0, i)}=\sum_{n=1}^{m} P_{n}\left(1-\alpha_{(0, i)}\right)^{n} .
$$

\section{B. Performance with relaying}

In this section, we extend the Markov model to the relaying case through the RSU for the packets of the platoon leader. Whenever the leader sends a packet to its platoon, this packet can be also received by the RSU closest to the leader, which then relays the packet as a broadcast. A loss for a broadcast link occurs here only if both the direct $(0, i)$ link and the relaying link fail, increasing the robustness of the system. The relaying link is composed of two links $(0, R S U)$ and $(R S U, i)$ as shown in Fig. 1. In other words, the final probability of loss between the leader and the vehicle $i$ in the presence of RSU is

$$
L_{i}=L_{(0, i)} \cdot L_{(\text {relay }, i)}
$$

where $L_{(0, i)}$ the loss of the direct link, computed in equation (16). $L_{\text {relay }, i}$ is the probability of loss of the relay link, corresponding to a loss on either the uplink or downlink, as shown in Fig. 1:

$$
L_{(\text {relay }, i)}=L_{(0, R S U)}+L_{(R S U, i)}-L_{(0, R S U)} \cdot L_{(R S U, i)}
$$

The loss on the relay link depends on the considered relaying technology and the associated spectrum.

1) Licensed spectrum: When using licensed spectrum, the relay link is collision exempt, as transmissions are scheduled. Therefore in (18), $L_{(0, R S U)}=f_{0,(0, R S U)}$ and $L_{(R S U, i)}=f_{0,(R S U, i)}$ are the probabilities of losses with no collision.

2) Unlicensed spectrum: The RSU in this case is a node like the others in the IEEE 802.11p system. The RSU here overhears the transmission on the leader-follower link, and its probability of loss is computed as in equation (16), taking into consideration in $\alpha_{(0, R S U)}$ the PER on the uplink of the relay.

If the packet is correctly decoded, the RSU is able to broadcast it, only once, to the other platoon members. We model this downlink of the relay by a one-stage Markov chain (like the one in Fig. 2, with $m=1$ ). The loss on the downlink is thus given by

$$
L_{(R S U, i)}=p_{c} f_{c,(R S U, i)}+\left(1-p_{c}\right) f_{0,(R S U, i)} .
$$

\section{Control and Communication Platooning INTERACTION AND PERFORMANCE}

The objective of this section is to detail the interaction between the proposed dynamic controller and the communication schemes in a realistic platooning simulation.

\section{A. Simulation environment description}

An overview of the system diagram is given in Fig. 4. We used the Simulink environment to model the vehicle dynamics and to implement the control law. Therefore, it is responsible to give periodic snapshots of the positions of the platoon vehicles to the communication simulator. As shown in Fig. 4, the communication framework is implemented

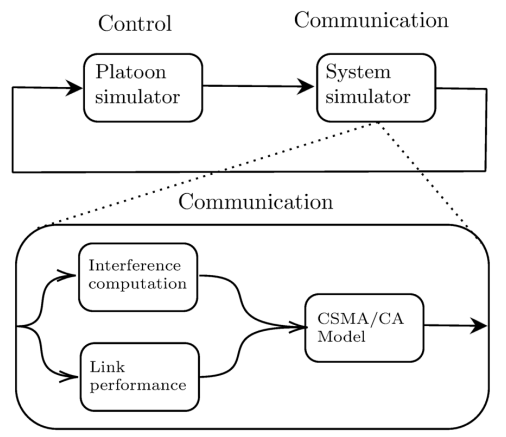

\begin{tabular}{|c|c|c|c|}
\hline \multicolumn{2}{|c|}{ Communication } & \multicolumn{2}{|c|}{ Controller } \\
\hline Parameter & Value & Parameter & Value \\
\hline Simulation & & PCACC & \\
\hline Duration & $1200 \mathrm{~s}$ & Leader factor $(C)$ & 0.5 \\
\hline Jammer profile & Check [6] & Desired distance $\left(D_{d e s}\right)$ & Adaptive \\
\hline Application & & Damping ratio $(\xi)$ & 2 \\
\hline CAM size & 500 bytes & Bandwidth $\left(\omega_{n}\right)$ & $0.5 \mathrm{~Hz}$ \\
\hline CAM interval & $100 \mathrm{~ms}$ & Vehicle \& Highway & \\
\hline PHY layer & & Actuator lag $(\tau)$ & $0.5 \mathrm{~s}$ \\
\hline Path loss & Winner+B1 LOS & Vehicle length $(L)$ & $16.5 \mathrm{~m}$ \\
\hline Noise power & $-174 \mathrm{dBm} / \mathrm{Hz}$ & Max. acceleration & $+2 \mathrm{~m} / \mathrm{s}^{2}$ \\
\hline Tx power & $22.5 \mathrm{dBm}$ & Min. acceleration & $-3 \mathrm{~m} / \mathrm{s}^{2}$ \\
\hline MCS & QPSK, R=1/2 & Radar interval & $60 \mathrm{~ms}$ \\
\hline Channel & Highway LOS & Lanes per direction & 2 \\
\hline Frequency & $5.9 \mathrm{GHz}$ & Lane width & $5 \mathrm{~m}$ \\
\hline Bandwidth & $10 \mathrm{MHz}$ & Max. traffic density $(\mathrm{M})$ & $100 \mathrm{car} / \mathrm{km} / \mathrm{lane}$ \\
\hline
\end{tabular}

Fig. 4. System diagram with control and communication interaction.

TABLE I

COMmunicATION AND CONTROLLER PARAMETERS

in Matlab and some main functions are highlighted at the bottom. The first one ("Interference computation") is responsible for computing the SNR (Signal to Noise Radio) and the SINR on the different links (V2V, downlink and uplink relay), provided that the packet is transmitted alone or is subject to collision with another packet. The second function ("Link performance") calculates the average PER for a given link quality (SNR/SINR) using the WLAN Toolbox of MATLAB, for the communication parameters of Table I. Lastly, the channel access to the medium is implemented as modeled using the Markovian model presented above, considering that vehicles broadcast a 500 bytes message each $100 \mathrm{~ms}$ in average. Note that for the link $(0, i)$, we assume a shadowing that increases linearly with the number of vehicles in the platoon $(1 \mathrm{~dB}$ per intermediate vehicle). As for the CSMA parameters, we have adopted $W_{0}=30$ and $m=1$ as the content window size and the retransmission attempts, respectively. Furthermore, for the relays schemes, we have implemented one RSU each one kilometer.

As for the control parameters, the platoon members are equipped with the proposed dynamic controller. The control strategy demands relative position and longitudinal velocity of the preceding vehicle so we assumed that the measurements are sampled each $60 \mathrm{~ms}$ with $1 \mathrm{~ms}$ delay and done by a long-range radar. Simulations are performed with a platoon size of $N=21$, with a jammer profile as the pattern from [6], but repeated 50 times. The maximum road traffic density that generates external interference to the platoon was considered as $M=100$ cars $/ \mathrm{km} / \mathrm{lane}$.

\section{B. Communication system performance}

We next describe the performance from a communication perspective. We present a communication radio link com- 
TABLE II

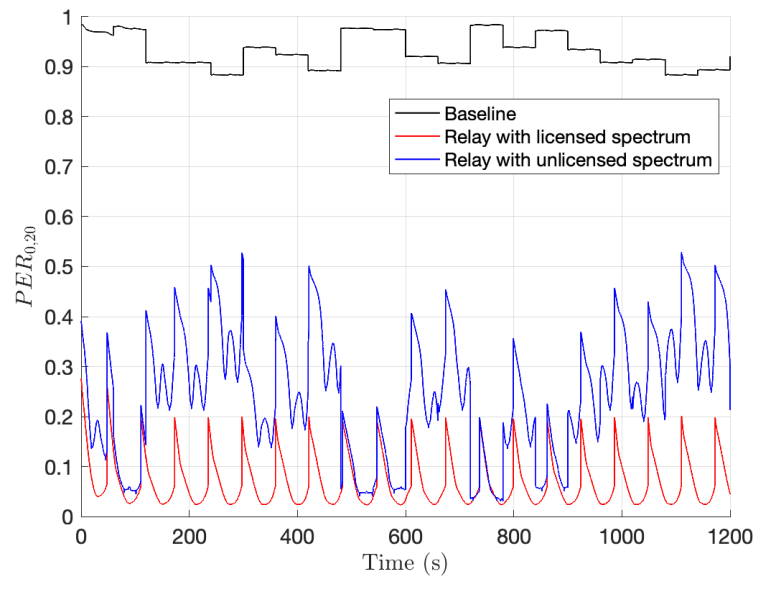

Fig. 5. PER for 20th vehicle for the different communication scenarios.

parison as shown in Fig. 5, where we present the average probability of loss between the leader and the last vehicle $(i=20)$ over time for each communication approaches considered. We observe from Fig. 5 that V2V scenario without any V2I results in a very high loss rate when compared to the relaying schemes. This loss is expected to be even higher for larger platoons. With relaying, a better quality link is observed when using licensed spectrum, due to the scheduled mode. Having a closer look on the curves of Fig. 5, the baseline performance fluctuates due to the change of the number of interfering vehicles with time. However, relay schemes exhibit a more cyclic behavior due to the evolution of the relative distance of the platoon with respect to the closest RSU from the leader vehicle (recall that a RSU is deployed every $1 \mathrm{Km}$ ). Our objective to ensure that the worst packet error rate in the platoon is below 0.25 in the presence of RSUs, which has been observed to result in a smooth control performance and spacing of the platoon. The impact of the RSU density in this scenario is indeed an interesting avenue for future research.

\section{Optimization of the average inter-vehicular spacing}

We now move to the evaluation of the robustness of our dynamic control scheme under different communication links. To this aim, we have adopted the inter-vehicle distance as the end-service performance metric. We apply the zeroorder hold mechanism as the holding strategy for the control signal during the periods of packet losses. In all simulations, we focus on minimizing the inter-vehicular distance in respect with a fixed value of all the other control parameters while ensuring that zero vehicles collisions occur. Note that we implemented a safety gap distance of $1 \mathrm{~m}$ for the emergency braking actuation to avoid collisions in practical settings.

We present the Table II the average inter-vehicular distance for 20 vehicle platoon over all communication approaches considered. Clearly, the V2V only scheme imposes higher inter-vehicular distances in the platoon, as much as $36 \mathrm{~m}$, when compared to relayed schemes where the average distance is around $1.7 \mathrm{~m}$. We also illustrate in Table II the minimum observed distance during the simulation (that must not go below $1 \mathrm{~m}$ to avoid emergency breaking) and the maximal observed distance on the platoon.

When looking at the platoon performance with relaying, very similar performance are observed for both relay
PERFORMANCE METRICS OVER DIFFERENT COMMUNICATION SCHEMES.

\begin{tabular}{c|ccc}
\hline \hline Outputs & Baseline & Relay licensed & Relay unlicensed \\
\hline \hline Avg. Dist. (m) & 36.7722 & 1.6467 & 1.7342 \\
Min. Obs. Dist. (m) & 1.0323 & 1.1195 & 1.2494 \\
Max. Obs. Dist. (m) & 64.0602 & 2.0479 & 2.1943 \\
Avg. PER (10th car) & 0.5294 & 0.0123 & 0.0584 \\
Avg. PER (20th car) & 0.9839 & 0.0814 & 0.2490 \\
\hline \hline
\end{tabular}

schemes with values around $6 \%$ different, despite the large difference in the loss rates, as recalled in the last rows of Table II. This similarity raises a question related to the necessity or not of licensed spectrum for platooning systems. This fact can be explained by the robustness of the dynamic PCACC control for a sampling rate of $100 \mathrm{~ms}$ under moderate packet losses.

\section{COnCluding Remarks}

In this paper, we have investigated the interplay between vehicular communication approaches and platooning performance. We proposed an analytical model to compute the probability of packet loss in a platoon with and without a relay support through RSU. An offline optimization of the control parameters is then presented based on the computed loss rate. Extensive numerical simulations show that the relaying scheme with licensed spectrum result in the lowest packet loss rate among the considered communication schemes. However, the best compromise between network cost (economic) and platoon performance is achieved when the RSUs are used over an unlicensed spectrum, due to the robustness of the proposed control scheme under sufficiently small communication errors.

\section{REFERENCES}

[1] G. Bianchi, "Performance Analysis of the IEEE 802.11 Distributed Coordination Function", IEEE Journal on Selected Areas in Communications, vol. 18 , no. 8, 2000, pp. 535-547.

[2] F. Marzouk, J. Rodriguez, and A. Radwan "Analysis and Enhancement of Platoon Management in Smart City", Proc. IEEE International Wireless Communications and Mobile Computing Conference, IWCMC, 2018.

[3] J. Fu, G. Wu, and R. Li, "Performance Analysis of Sidelink Relay in SCMA-based Multicasting for Platooning in V2X", IEEE International Conference on Communications Workshops, 2020.

[4] D. Jia, R. Zhang, K. Lu, J. Wang, Z. Bi, and J. Lei, "Improving the Uplink Performance of Drive-Thru Internet via Platoon-Based Cooperative Retransmission," IEEE Transactions on Vehicular Technology, vol. 63, no. 9, 2014, pp. 4536-4545.

[5] A. Vinel, L. Lan, and N. Lyamin, "Vehicle-to-vehicle communication in C-ACC/platooning scenarios," IEEE Communications Magazine, vol. 53, no. 8, 2015, pp. 192-197.

[6] V. Vukadinovic, et al., "3GPP C-V2X and IEEE $802.11 \mathrm{p}$ for Vehicleto-Vehicle communications in highway platooning scenarios," Ad Hoc Networks, vol. 74, 2018, pp. 17-29.

[7] J. Ploeg, E. Semsar-Kazerooni, G. Lijster, N. van de Wouw, and H. Nijmeijer, "Graceful degradation of cooperative adaptive cruise control." IEEE Transactions on Intelligent Transportation Systems, vol. 16 , no. 1,2015 , pp. $488-497$.

[8] P. Fernandes, and U. Nunes, "Platooning with IVC-enabled autonomous vehicles: Strategies to mitigate communication delays, improve safety and traffic flow", IEEE Transactions on Intelligent Transportation Systems, vol. 13, no. 1, 2012.

[9] T. R. Gonçalves, V. S. Varma, and S. E. Elayoubi, "Vehicle platooning schemes considering V2V communications: a joint communication/control approach," Proc. IEEE Wireless Communications and Networking Conference, WCNC 2020.

[10] M. Sybis, et al., "Communication Aspects of a Modified Cooperative Adaptive Cruise Control Algorithm", IEEE Transactions on Intelligent Transportation Systems, 2019.

[11] R. Rajamani. Vehicle dynamics and control, Springer Science \& Business Media, 2011 